\title{
EFFECTS OF DESPECKLING ON THE ESTIMATION OF FRACTAL DIMENSION FROM SAR IMAGES
}

\author{
Gerardo Di Martino, Giovanni Poggi, Daniele Riccio, Luisa Verdoliva \\ Università di Napoli Federico II, Via Claudio 21, 80125, Napoli, Italy \\ \{gerardo.dimartino,poggi, daniele.riccio,verdoliv\}@unina.it
}

\begin{abstract}
The estimation of the fractal dimension of a natural surface using spectral analysis of amplitude SAR data is a powerful tool for geophysical applications. However, the obtained estimates are influenced by the presence of speckle. In this paper we present first results regarding the analysis of the effects on fractal dimension maps due to the application of despeckling techniques on SAR images. The use of simulated data allows to obtain also multilook images, presenting a very high number of looks, which should represent a virtually speckle-free image. In the results section different despeckling techniques are considered, relaying on both classical spatial filtering and non-local means. The presented results allow to draw meaningful conclusions on the effects of speckle and despeckling on spectral estimation and fractal dimension retrieving.
\end{abstract}

Index Terms - SAR, fractals, speckle reduction, spectral estimation.

\section{INTRODUCTION}

The development of automatic tools for SAR data interpretation is a very relevant issue in the remote sensing community. The availability of value-added SAR products, providing a quantitative description of physical properties of the observed surfaces, is a key factor for broadening the community of end-users interested in SAR applications. In particular, estimating the fractal dimension of surfaces from SAR images holds a great interest for geophysicists and geologists, as this parameter provides a meaningful quantitative measure of the roughness of natural surfaces, preferred to classical statistical descriptors such as the standard deviation and the correlation length of the height process [1]-[3].

In [4] some of these authors introduced new models for the imaging of natural surfaces and discussed results regarding the estimation of the fractal dimension from a single amplitude-only SAR image. The proposed technique relies on spectral estimation, performed using an advanced non-parametric spectral estimator. The method proposed in [4] is able to provide, as output, a new image, the fractal dimension map, which gives for each point the locally estimated fractal dimension of the imaged surface. The technique has been applied to actual SAR images in [5] where considerable efforts were devoted to properly take into account the presence of speckle, inherent of any SAR image, and limit its potentially detrimental effects.

In this paper we carry out a deeper investigation of the effects of speckle on fractal dimension estimation and analyze the effects due to the application of different despeckling techniques. We borrow concepts from [6] where a new methodology for studying the effects of despeckling techniques was proposed. In particular, by feeding the SARAS simulator [7] with fractal DEMs synthesized using the Weierstrass-Mandelbrot function [4] [5], we simulate SAR images relevant to surfaces presenting different fractal dimensions. Hence, following the approach proposed in [6], we generate a large number of images sharing the same input DEM, and average them so as to obtain a multilooked version with an arbitrary number of looks. By doing so, we approximate with arbitrary precision the ideal infinite-look SAR image, in which all speckle contributions are washed out. By comparing the fractal maps obtained with the singlelook realizations and with the multilook reference image we are therefore able to investigate the effects of speckle on fractal dimension estimation.

The results obtained with the speckle-free image can be expected to represent an upper bound for the performance obtained with of single-look images and can help assessing the estimation procedure itself and guide its further development. In addition, by means of an end-to-end evaluation of performance, we study the effects of a preliminary despeckling pass on the quality of the fractal dimension estimation, so as to understand if the use of these techniques is appropriate for this task. A more general evaluation of the impact of speckle and despeckling on spectral estimation is presented in the final section of the paper.

\section{EXPERIMENTAL FRAMEWORK}

In this section we briefly describe the considered experimental framework, focusing first on the simulation procedure and then on the fractal dimension map generation. 

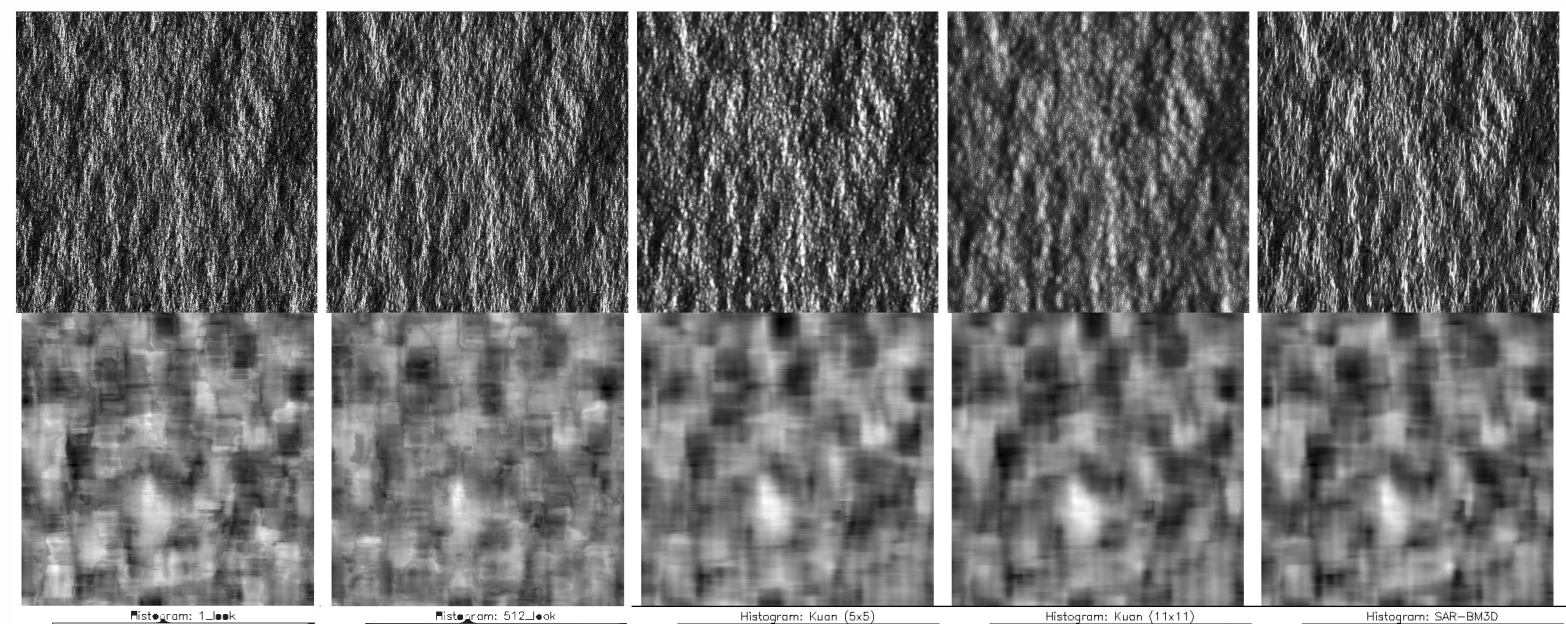

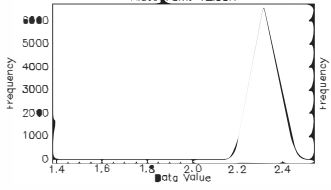

(a)

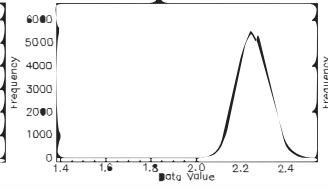

(b)

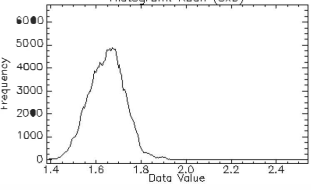

(c)

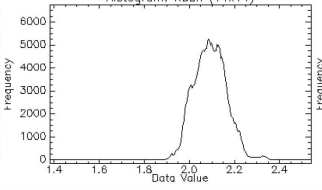

(d)

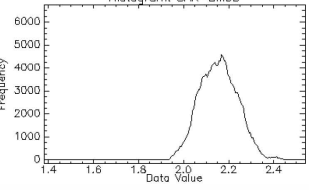

(e)

Fig. 1 From top to bottom: simulated images ( $D=2.3$ ), fractal dimension maps and their histograms; (a) 1 look; (b) 512 look; (c) Kuan 5x5; (d) Kuan 11x11; (e) SAR-BM3D.

\subsection{Simulation approach}

The proposed simulator is based on geometrical and electromagnetic models, used for the evaluation of the reflectivity function of the scene, and on a model for the transfer function of the system, which is used for the evaluation of the SAR raw signal [7]. It requires as input a Digital Elevation Model (DEM), the roughness of the resolution cell, the electromagnetic parameters of the surface and radar and orbital data of the sensor. In this work we provide as input to the simulator fractal DEMs synthesized using the Weierstrass-Mandelbrot function with different values of the fractal dimension [4]-[5].

The simulation procedure allows also the definition of virtually speckle-free images [6]. In fact, thanks to the use of the simulator, we can obtain a SAR image presenting a finite but very large number of looks, approximating with arbitrary precision the infinite look image. This is obtained computing the average of a large number of independent images relevant to the same input DEM. In particular, in this paper the speckle-free reference images are obtained by averaging 512 independent intensity images.

\subsection{Fractal dimension map estimation}

The fractal dimension estimation is performed through spectral analysis of the amplitude SAR data: all relevant details can be found in [4]. In particular, the Capon spectral estimator is used, due to its effectiveness in the estimation of power-law spectra [8]. A linear regression is then performed on the estimated spectrum in order to obtain the fractal dimension of the observed surface. Due to the aliasing and to the presence of speckle, the range of wavenumbers considered for fractal dimension estimation is limited both in the low and high wavenumbers regions [4]. Moreover, in order to obtain a point-by-point map of the fractal dimension the spectral estimation is performed using a window sliding over the whole SAR image: the window encloses small portions of the image that are supposed to share the same fractal parameters, and a unique value of fractal dimension is assigned to the center pixel of each window. The dimension of the sliding window results from a trade-off between the accuracy of the estimation, the resolution required for the generated fractal maps and the spatial scale of the surface features we are interested in [4]-[5]. In the following, we present results only for the window size $(51 \times 51)$ and wavenumber range used in previous works.

\section{RESULTS}

In this section we present a discussion of preliminary results obtained on a limited set of case studies. We consider two test images obtained by providing as input to the simulator synthetic fractal DEMs with fractal dimension $D=2.3$ and $D=2.2$. Moreover, we consider two despeckling techniques, one relying on the linear filtering approach (Kuan filter [9]) and the other one on non-local filtering (SAR-BM3D [10], 

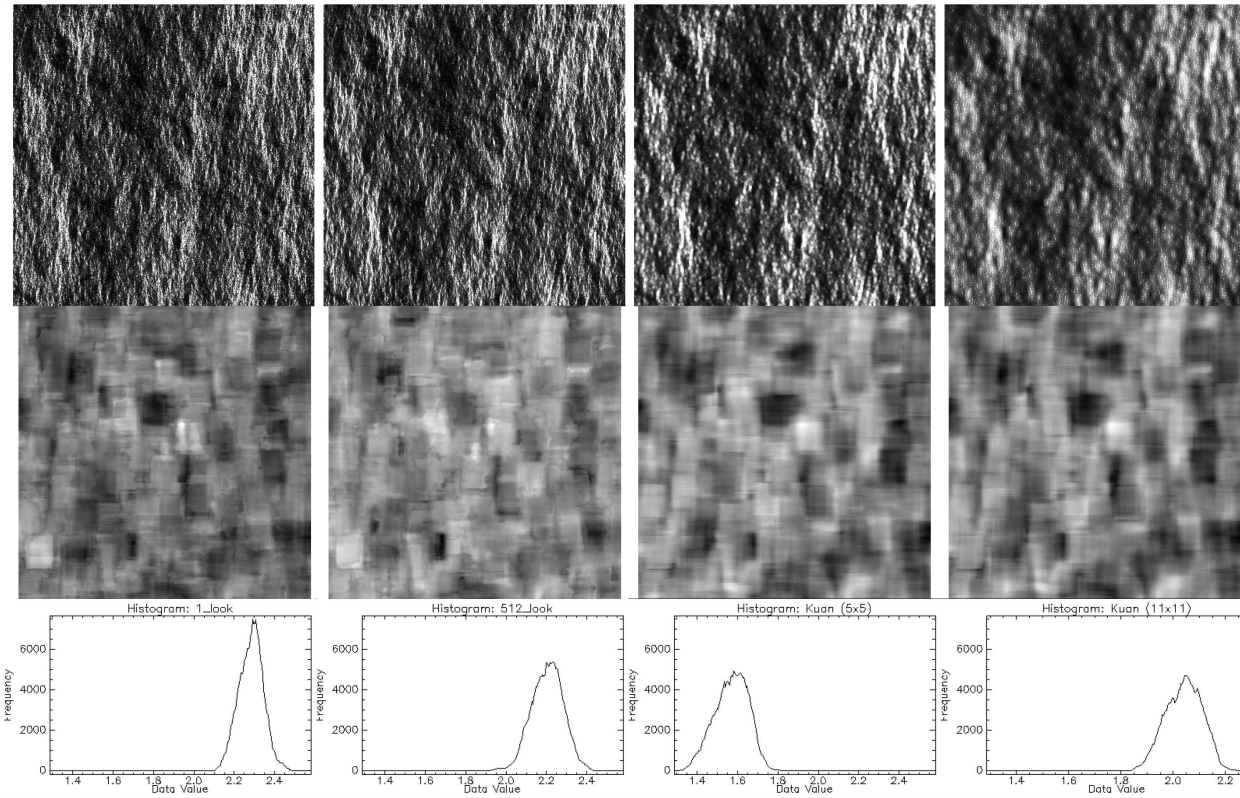

(a)

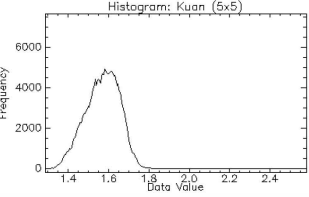

(c)

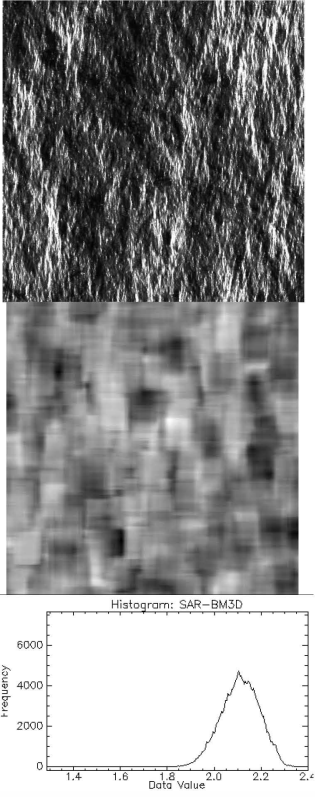

(e)

Fig. 2 From top to bottom: simulated images $(D=2.2)$, fractal dimension maps and their histograms; (a) 1 look; (b) 512 look; (c) Kuan 5x5; (d) Kuan 11x11; (e) SAR-BM3D.

[11]). In order to provide some hints on the relevant role of filter parameter tuning, for the Kuan filter we show results obtained with two window sizes, $5 \times 5$ and $11 \times 11$ pixels.

In the first line of Figs. 1 and 2 we show $512 \times 512$ sections of the images relevant to the cases $D=2.3$ and $D=2.2$, respectively: namely, the first realization of the simulated image, the 512-look reference image and the filtered images. Typical parameters of the Cosmo-SkyMed sensor were set for the simulation. Starting from these images, the fractal dimension maps reported in the second line of Figs. 1 and 2 were estimated, characterized by the histograms reported in the third line. The value of the mean and standard deviation of the maps are reported in Tab. I.

Observing the values in Tab. I and the histograms in Fig. 1 some meaningful conclusions can be drawn. With regard to the 512 look images, we observe in general a decrease in the average fractal dimension and an increase in the standard deviation: in the case of $D=2.2$ this decrease imply an estimated value closer to the expected one, while for $D=2.3$ the estimate gets worse. Probably, in the 512 look image the speckle component is, in fact, strongly attenuated, but we may face the problem of the emergence of the topographic component related to the specific surface realization used in the simulation. As a matter of fact, this component can significantly depart from the imposed ideal fractal behavior. However, this topic is out of the scope of the present paper, and will be object of future research.

With regard to the maps obtained from the filtered images, the most interesting fact is that performance is significantly impaired, with a strong underestimation of the average fractal dimension. This negative result, however, prompted us to perform a deeper and more general investigation of the effects of despeckling on spectral estimation. For this reason, we estimated the power spectral densities of all the simulated images along the range direction using all the available samples on the $512 \times 512$ sections. The graphs of the obtained PSDs are reported in Figs. 3 and 4 for $D=2.3$ and $D=2.2$, respectively.

The strong impact of despeckling on the image spectra is evident just looking at the figures: in particular, both the Kuan filters heavily distort the image spectral behavior, while SAR-BM3D causes not so evident effects. In general the low-pass filtering characteristics of the filters dictate a significant attenuation of the image high frequency components, filtering out also signal components. This behavior can be partly appreciated also for the 512 look image. The two vertical axes reported in the figures highlight the frequency range involved in the estimation of the fractal dimension maps, which are estimated using image patches enclosed in the $51 \times 51$ sliding windows, thus implying the use of higher frequencies only. The highlighted region is relevant to the spectrum area most affected by distortions. Whenever fractal dimension estimation is applied on these spectra, it will result in very inaccurate estimates: in the Kuan cases not even the spectrum linear behavior in the log-log plane is retained, while for SAR$\mathrm{BM} 3 \mathrm{D}$ the behavior of the spectrum is almost linear, but presenting a slope significantly higher than the expected one. 


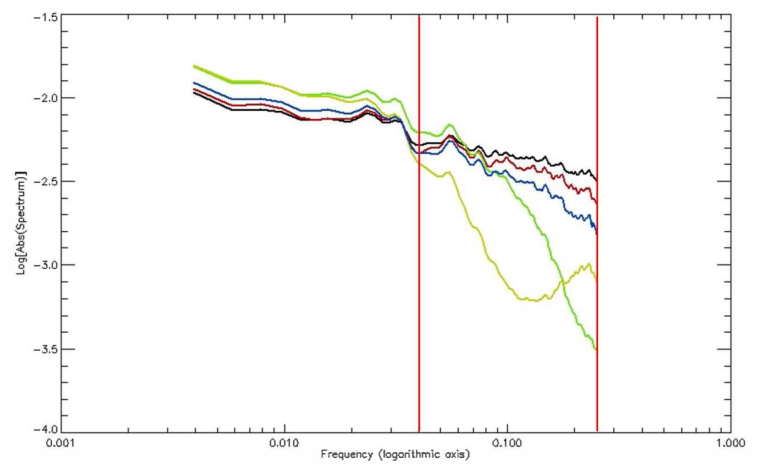

Fig. 3 Estimated spectra for $D=2.3: 1$ look (black); 512 look (dark red); Kuan 5x5 (green); Kuan $11 \times 11$ (cyan); SAR-BM3D (blue).

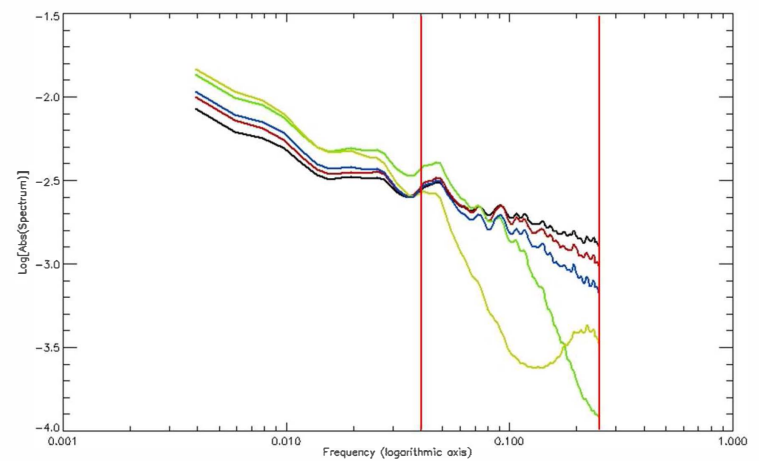

Fig. 4 Estimated spectra for $D=2.2: 1$ look (black); 512 look (dark red); Kuan 5x5 (green); Kuan 1 1x11 (cyan); SAR-BM3D (blue).

\section{CONCLUSIONS}

In this paper we presented first results regarding the analysis of the effects of speckle and of the application of despeckling techniques on the estimation of fractal dimension maps from amplitude SAR data. The obtained results highlighted the heavy impact of despeckling on the spectral behavior of the considered simulated images. The observed effects range from heavy distortions (responsible for the departure of the spectra from the expected linear behavior in the log-log plane) to excessive attenuation of image high frequency content.

Given these results, at the moment, the estimation of the fractal dimension maps should be implemented starting from single look images, and cutting out high frequency components, affected by both speckle and aliasing noise. Further research is necessary to explore the impact of despeckling techniques on spectral estimation and, in particular, to better characterize the spatial scales affected by the filtering.
Table I Statistics of the fractal dimension maps

\begin{tabular}{|c|c|c|c|c|}
\hline & $\begin{array}{c}\text { Mean } \\
(D=2.3)\end{array}$ & $\begin{array}{c}\text { St. dev. } \\
(D=2.3)\end{array}$ & $\begin{array}{c}\text { Mean } \\
(D=2.2)\end{array}$ & $\begin{array}{c}\text { St. dev. } \\
(D=2.2)\end{array}$ \\
\hline 1 look & 2.32 & 0.06 & 2.29 & 0.06 \\
\hline 512 look & 2.26 & 0.07 & 2.21 & 0.08 \\
\hline Kuan (5x5) & 1.65 & 0.08 & 1.57 & 0.08 \\
\hline Kuan (11x11) & 2.10 & 0.07 & 2.04 & 0.07 \\
\hline SAR-BM3D & 2.16 & 0.08 & 2.11 & 0.08 \\
\hline
\end{tabular}

\section{REFERENCES}

[1] B. B. Mandelbrot, The Fractal Geometry of Nature. New York: Freeman, 1983.

[2] G. Franceschetti, D. Riccio, Scattering, Natural Surfaces and Fractals. Burlington, MA: Academic Press, 2007.

[3] B.A. Campbell, "Scale-dependent surface roughness behavior and its impact on empirical models for radar backscatter", IEEE Trans. Geosci. Remote Sens., vol. 47, no. 10, pp. 3480-3488, Oct. 2009.

[4] G. Di Martino, D. Riccio, and I. Zinno, "SAR Imaging of Fractal Surfaces", IEEE Trans. Geosci. Remote Sens., vol. 50, no. 2, pp. 630-644, Feb. 2012.

[5] G. Di Martino, A. Iodice, D. Riccio, G. Ruello, and I. Zinno, "On the fractal nature of volcano morphology detected via SAR image analysis: the case of Somma-Vesuvius Volcanic Complex", European Journal of Remote Sensing, no. 45, pp. 177-187, 2012.

[6] G. Di Martino, M. Poderico, G. Poggi, D. Riccio, and L. Verdoliva, "Benchmarking framework for SAR despeckling", IEEE Trans. Geosci. Remote Sens., 2013 in print, available on IEEEXplore.

[7] G. Franceschetti, M. Migliaccio, D. Riccio, and G. Schirinzi, "SARAS: a SAR raw signal simulator," IEEE Trans. Geosci. Remote Sens., vol. 30, no. 1, pp. 110-123, Jan. 1992.

[8] S. M. Kay, Modern Spectral Analysis. Englewood Cliffs, NJ: Patience Hall, 1999.

[9] D. T. Kuan, A. A. Sawchuk, T. C. Strand, and P. Chavel, "Adaptive Noise Smoothing Filter for Images with SignalDependent Noise," IEEE Trans. Pattern Anal. Machine Intell., vol. PAMI-7, no. 2, pp. 165-177, Mar. 1985.

[10] S. Parrilli, M. Poderico, C. V. Angelino, and L. Verdoliva, "A nonlocal SAR image denoising algorithm based on LLMMSE wavelet shrinkage," IEEE Trans. Geosci. Remote Sens., vol. 50, no. 2, pp. 606-616, Feb. 2012.

[11] D. Cozzolino, S. Parrilli, G. Scarpa, G. Poggi, L. Verdoliva, "Fast Adaptive Nonlocal SAR Despeckling", IEEE Geosci. Remote Sens. Lett., 2013 to be published. 